\title{
The Meteorological Disaster Risk Assessment Based on the Diffusion Mechanism
}

\author{
Guo Shujun \\ Hebei Province Meteorological Bureau, Shijiazhuang 050021, China \\ E-mail:gsjun888@sina.com \\ Received 9 September 2011 \\ Accepted 27 April 2012
}

\begin{abstract}
At present, the study on meteorological disasters risk is mostly for single hazard-affected body. With the economic and social development in recent years, meteorological disaster risk is appearing more "system" characteristics. Meteorological disasters not only impact on direct hazard-affected bodies, but also on other bodies through the direct victims. In general, the influence of the direct victims transmits to other bodies in some diffusion ways, which is rather different from the disaster chain, which we well known. In this paper, we propose the concept of diffusion of meteorological disaster risk, study the diffusion mechanism of the meteorological disaster risk, make a simulation system of risk diffusion analysis, and provide the analysis method and the calculation model for more reasonably and more comprehensively understanding the diffusion of meteorological disaster risk. Taking blizzard of North China in early winter 2009 as an example, we construct a risk diffusion network of the low temperature and blizzard disaster, systemically analyze what would be affected by the disaster, and give the scene expression of risk with prompt function, Which is useful for government and relevant departments to make the decisions in dealing with meteorological disaster risk.
\end{abstract}

Keywords: meteorological disaster, risk diffusion, diffusion network, simulation

\section{基于风险扩散机制的气象灾害风险评估}

\author{
郭树军 \\ 河北省气象局，石家庄 050021
}

\begin{abstract}
摘要：目前有关气象灾害风险的研究, 大多是针对单承灾体的风险分析。随着经济社会的发展, 近年来气 象灾害风险呈现出 “系统型” 的特点。气象灾害不仅对直接承灾体产生影响, 还通过直接承灾体对其它相 关的承灾体产生影响, 通常以扩散方式传导, 与人们熟知的灾害链并不一样, 相关研究较少。本文提出了 气象灾害风险扩散的概念, 揭示了气象灾害风险的扩散机理, 建立了风险扩散分析的仿真模拟系统, 为较 正确和更全面地认识气象灾害风险扩散现象提供了分析方法和计算模型。以 2009 年华北初冬暴雪为例进行 了风险扩散的过程分析, 建立了低温暴雪灾害的风险扩散图, 对低温暴雪灾害可能影响的主要领域进行了 系统分析, 给出了具有风险预警提示的情景表示, 为政府和有关部门应对气象灾害风险提供了决策依据。
\end{abstract}

关键词: 气象灾害, 风险扩散, 扩散网络, 仿真模拟

\section{1. 引言}

按照世界气象组织的统计, 气象灾害约占自然 灾害的 70\%左右。随着经济社会的发展, 气象灾害 的连锁效应显著, 连锁效应造成的影响有时会比灾 害本身造成的损失还要大, 气象灾害风险存在扩散 效应 ${ }^{[1-3]}$ 。目前的气象灾害风险研究虽然取得了不少 的进展, 但是, 国内外大部分气象灾害的风险评价 工作都是基于 “风险度=危险度 $\times$ 易损度” 这一传统 的风险评价体系 ${ }^{[4-7]}$, 该评价体系主要针对单一承灾
体, 并不能完全解释气象灾害风险在经济社会领域 中的扩散效应。

近年来, 众多科学研究机构启动了综合风险的 相关研究计划, 国际科学联盟 (ICSU) 2008 年启动 了灾害风险综合研究的计划（IRDR），提出在风险 和减轻灾害风险研究中, 要对各种灾害或灾害链进 行多学科、多尺度的综合性深入研究 ${ }^{[8]}$ 。2009 年 IHDP 启动了新一轮综合风险防范 (IHDP-IRG) 的科 学研究计划, 重点强调了灾害链风险评价模型的改进 和灾害风险情景模拟工具的完善。有学者利用基于 
宏观经济学的投入产出关系研究灾害的宏观效应 ${ }^{[9}$ ${ }^{10]}$ 。但是, 针对风险扩散机制的研究及应用较为鲜 见。

本文拟从风险产生的关键环节着手, 进行气象 灾害风险扩散机理的研究, 揭示气象灾害风险产生 和发展、扩散的全过程, 为政府和风险管理相关部 门全面认识与有效管理风险提供理论依据和技术支 撑。

\section{2. 气象灾害风险扩散机理}

\section{1. 定义}

为了更好地描述气象灾害风险的扩散现象, 给 出以下定义。

定义 1 气象灾害风险是由气象因素导致人类社 会造成损失的一种未来情景。

该定义是基于黄崇福的自然灾害风险的情景定 义提出的气象灾害情景定义, 优点是为描述风险提 供了一个新的更加符合现实的表达方式, 为气象灾 害风险扩散的情景表示提供了依据。

定义 2 气象灾害风险扩散是指气象致灾力作用 于承灾体后, 由于经济社会的系统性和承灾体的关 联性，对其他承灾体产生的波及效应。

定义 3 风险节点是指受到气象致灾力直接作用 或间接作用的承灾体。

定义 4 气象灾害风险由一个风险节点向另一个 风险节点传播扩散的路径称为气象灾害风险扩散通 道。

定义 5 由风险扩散的起始点对直接承灾体产生 的扩散称为一级扩散, 由直接承灾体对与其它相关 的承灾体产生的扩散称为二级扩散, 以此类推, 构 成一个分层级的扩散结构体, 该结构体称为风险扩 散网络。

定义 6 由下级风险节点向上级风险节点进行的 扩散称为逆向风险扩散。

\section{2. 气象灾害风险扩散的拓扑结构分析}

根据气象灾害对相关领域的影响建立气象灾害 风险扩散物理模型, 不失一般性, 我们假设通过风 险专家建立了如下的气象灾害风险扩散物理模型, 如图 1 所示。

图中 $V_{1}$ 是风险的扩散源点, $V_{1}, V_{2} \cdots V_{7}$ 表示风险 的各个节点, 箭头表示风险的传递方向。例如: $V_{I}$ 到 $V_{2} 、 V_{5}$ 是风险的传递， $V_{5} 、 V_{1}$ 对 $V_{1}$ 有风险的逆向扩 散。下面给出风险扩散网络拓扑结构的层级分析算 法, 识别扩散网络的扩散和逆向扩散关系。

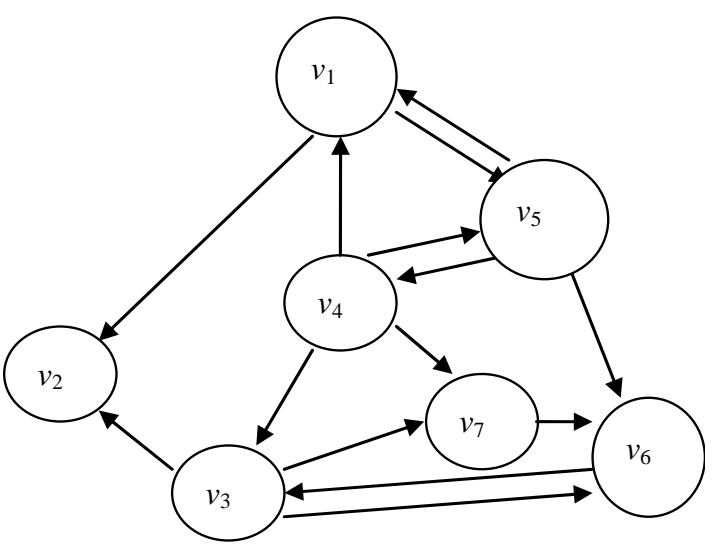

图 1. $v_{1}$ 作用于其它节点的气象灾害风险扩散与逆向 扩散物理模型

\section{1）节点连通路径信息矩阵}

根据风险节点间的关系, 建立一个 $\mathrm{n}$ 维信息矩

阵, $\mathrm{n}$ 表示风险扩散图的风险节点个数。表示为:

$$
A=\left(l_{i j}\right)_{n \times n}
$$

其中

$$
I_{i j}= \begin{cases}1, \text { 若从风险节点 } & v_{i} \text { 到 } v_{j} \text { 有通路 }\left(v_{i}, v_{j}\right) \text { 连接 } \\ 0, \text { 若从风险节点 } & v_{i} \text { 到 } v_{j} \text { 无通路 }\left(v_{i}, v_{j}\right) \text { 连接 }\end{cases}
$$

以图 1 为例, 我们得到节点连通路径信息矩阵如式 (2) 所示。

$$
\begin{aligned}
& \begin{array}{lllllll}
v_{1} & v_{2} & v_{3} & v_{4} & v_{5} & v_{6} & v_{7}
\end{array} \\
& A=\begin{array}{c}
v_{1} \\
v_{2} \\
v_{3} \\
v_{4} \\
v_{5} \\
v_{6} \\
v_{7}
\end{array}\left[\begin{array}{lllllll}
0 & 1 & 0 & 0 & 1 & 0 & 0 \\
0 & 0 & 0 & 0 & 0 & 0 & 0 \\
0 & 1 & 0 & 0 & 0 & 1 & 1 \\
1 & 0 & 1 & 0 & 1 & 0 & 1 \\
1 & 0 & 0 & 1 & 0 & 1 & 0 \\
0 & 0 & 1 & 0 & 0 & 0 & 0 \\
0 & 0 & 0 & 0 & 0 & 1 & 0
\end{array}\right]
\end{aligned}
$$

$A$ 可以看成由风险节点 $i$ 到风险节点 $j$ 是否有 直接扩散通路的描述。矩阵的第 $i$ 行表示节点 $i$ 到 所有节点有无扩散, 矩阵的 $j$ 列表示所有节点有没 有到节点 $j$ 的汇聚。

\section{2)风险扩散数据结构}

风险扩散网络结构可以用节点集合和节点间关 系集合来表示, $P S=\{D, R\}$, 其中, $P S$ 表示风险扩 散网络, $D$ 表示节点集合, $R$ 表示节点间关系集合。

为了将节点进行分级, 进一步将风险扩散网络 细分为分层级的节点集合和带方向的扩散关系集 
合, $P S=\{L, S R, N R\}$, 其中, $L$ 表示节点的层级集 合, $S R$ 表示风险扩散关系集合, $N R$ 表示风险逆向扩 散关系集合。

\section{3）确定风险扩散网络的层级结构}

遍历节点连通路径信息矩阵的第一行所有节

点, 选取受致灾力节点 $V_{l}$ 直接作用的节点, 将它们 建为一级扩散节点集合 $L_{l}$, 并将关系集合中的对应 关系移致 $S R$ 中。

以一级风险扩散节点集合 $L_{1}$ 的每个节点扫描信 息矩阵, 选取该节点直接作用的节点, 将它们加入 到二级扩散节点集合 $L_{2}$ 中, 如果节点已在上层级或 本层级中出现, 则剔除, 同时将关系集合中的对应 关系移致 $S R$ 中, 如果直接作用的节点出现在本级, 说明存在同级节点间的扩散, 扩散关系要移致 $S R$ 中。依次类推, 重复以上操作, 直至完成所有节点 的扫描, 最后, 在关系集合中保留的就是逆向扩散 关系集合 $N R$ 。

由此得到分层级的节点集合和分方向关系集 合。

$$
\begin{aligned}
& L_{0}=\left\{V_{1}\right\} \\
& L_{1}=\left\{V_{2}, V_{5}\right\} \\
& L_{2}=\left\{V_{1}, V_{6}\right\} \\
& L_{3}=\left\{V_{7}, V_{3}\right\} \\
& S R=\left\{r_{12}, r_{15}, r_{54}, r_{56}, r_{47}, r_{43}, r_{63}, r_{37}\right\} \\
& N R=\left\{r_{51}, r_{41}, r_{45}, r_{76}, r_{36}, r_{32}\right\}
\end{aligned}
$$

基于以上的分级, 建立的风险扩散网络拓扑结 构如图 2 所示。

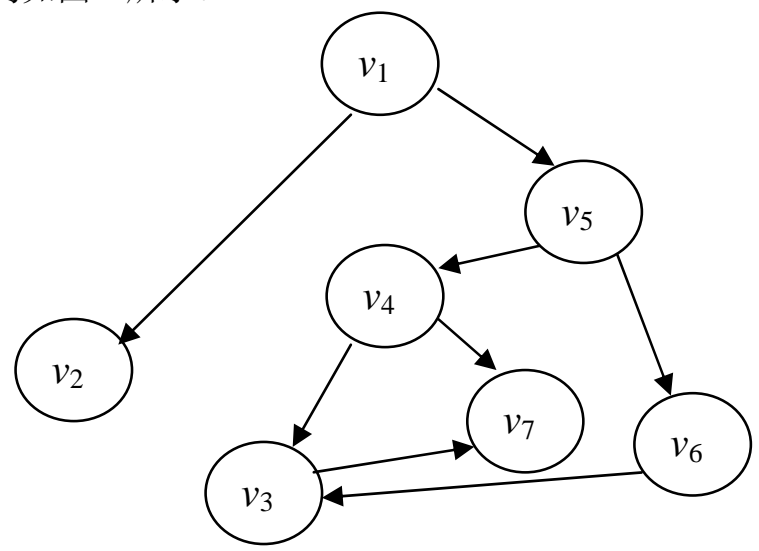

图 2. $v_{1}$ 作用于其它节点的气象灾害风险扩散模型

利用上述方法可以将风险扩散的物理网络转变 为由风险扩散和风险逆向扩散组成的逻辑分析结 构, 为风险扩散计算奠定了基础。同理, 按照逆向 风险扩散关系集合，建立的逆向风险扩散网络拓扑 结构如图 3 所示。

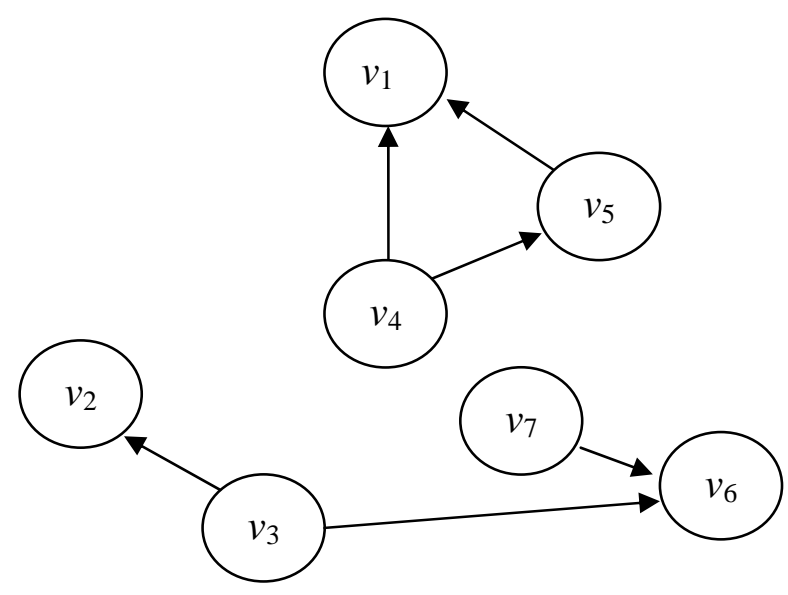

图 3. 逆向扩散模型

\section{3. 风险扩散的计算}

根据实际情况, 给出两类风险扩散的计算方 法:

\section{1) 概率风险扩散的计算}

上游节点风险乘以上下游节点间的风险扩散系 数得到上游节点对下游节点的风险扩散结果, 假设 节点 $i$ 是节点 $j$ 的上游节点, 那么由节点 $i$ 扩散给节 点 $j$ 的风险为:

$$
P_{j}=P_{i} \times S_{i \rightarrow j}
$$

其中, $P_{i}$ 为上游节点 $i$ 的风险值,

$S_{i \rightarrow j}$ 为节点 $i$ 对节点 $j$ 的风险扩散系数,

$P_{j}$ 为节点 $i$ 对节点 $j$ 的风险扩散结果。

\section{2) 模糊风险扩散的计算}

假设节点 $i$ 是节点 $j$ 的上游节点, 如果节点间风 险扩散系数是模糊集合，则风险扩散的计算采用下 面公式:

$$
P_{J}(j, p)=\sup _{i \in I}\left\{P_{I}(i, p) \wedge R(i, j)\right\}
$$

其中, $P_{I}(i, p)$ 为上游节点 $i$ 的模糊风险, $R(i, j)$ 为节点间的模糊系数, $P_{J}(j, p)$ 为节点 $i$ 对节点 $j$ 扩散的模糊风险。

\section{4. 节点的风险融合}

图 4 表示风险节点 $R$ 有多个来源的风险, 那么, 节点 $R$ 的综合风险值由各个来源的风险值, 按照给 定的权重相加求得。即:

$$
P=\sum_{i=1}^{n} w_{i} \times P_{i}
$$


式中, $w_{i}$ 表示权重, $P_{i}$ 表示各风险源的风险值。

如果各个来源的风险等级不一致, 则不能直接 融合。

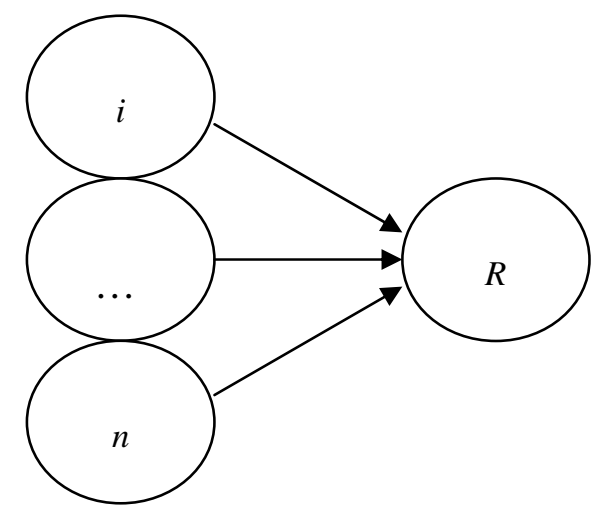

图 4. 多来源风险的融合

\section{3. 气象灾害风险扩散分析仿真系统}

根据风险扩散的基本原理, 利用面向对象的计 算机软件技术, 建立了气象灾害风险扩散分析仿真 系统（如图 5 所示）, 能够进行定量与定性相结合 的风险扩散分析。气象灾害风险扩散分析仿真系统 主要包括风险扩散网络构建、风险分析计算以及风 险的情景表示等功能。

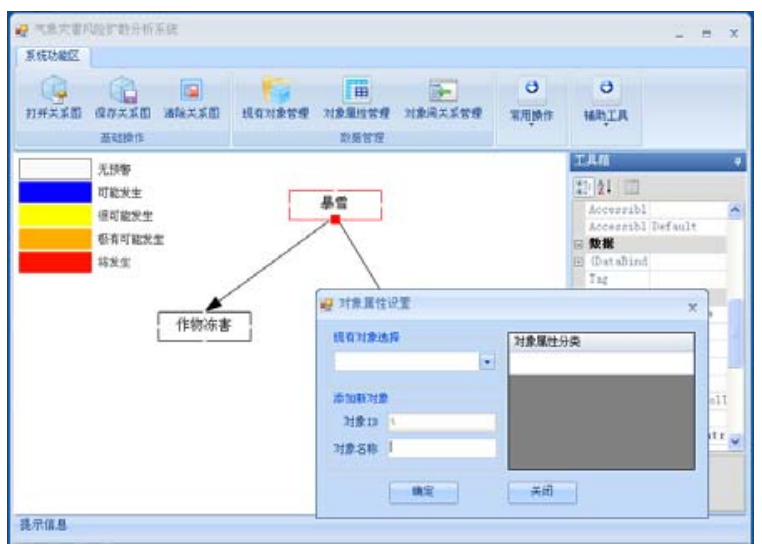

图 5. 风险分析仿真系统界面图

\section{4. 实例分析一以 2009 年华北暴雪灾害为例}

\section{1. 研究范围}

研究区域为石家庄地区, 研究的灾害为持续暴 雪, 研究的领域为区域内的农业、蔬菜损失风险及 其对相关领域的影响。

\section{2. 风险扩散的识别}

根据暴雪的灾害影响按照 “先面后点、先上后 下、横向交叉” 的分析原则, 进行灾害风险扩散的 识别, 建立暴雪灾害的风险扩散网络。

暴雪首先对大田作物冬小麦、设施蔬菜等农 业, 以及交通造成直接影响, 此为一级风险的扩 散。冬小麦将受到霜冻减产的威胁, 蔬菜大棚垮塌 对设施蔬菜的生产造成影响, 降雪使交通受阻直接 造成运输不畅, 此为暴雪的二级风险所在。蔬菜减 产和运输不畅会造成蔬菜涨价, 运输不畅会还造成 日常生活用品的供给不足, 引发涨价, 粮食是基础 性战略物资, 粮食的减产会给相关的产业造成影 响, 此为三级风险扩散。蔬菜、日用品涨价, 会给 中低收入的居民生活带来影响, 此为四级风险扩 散。建立的扩散网络如图 6 所示。

\section{3. 确立节点间的风险扩散关系}

节点间风险扩散关系的确立是进行风险扩散计 算的基础, 下面对风险扩散网络中的主要关系进行 说明。

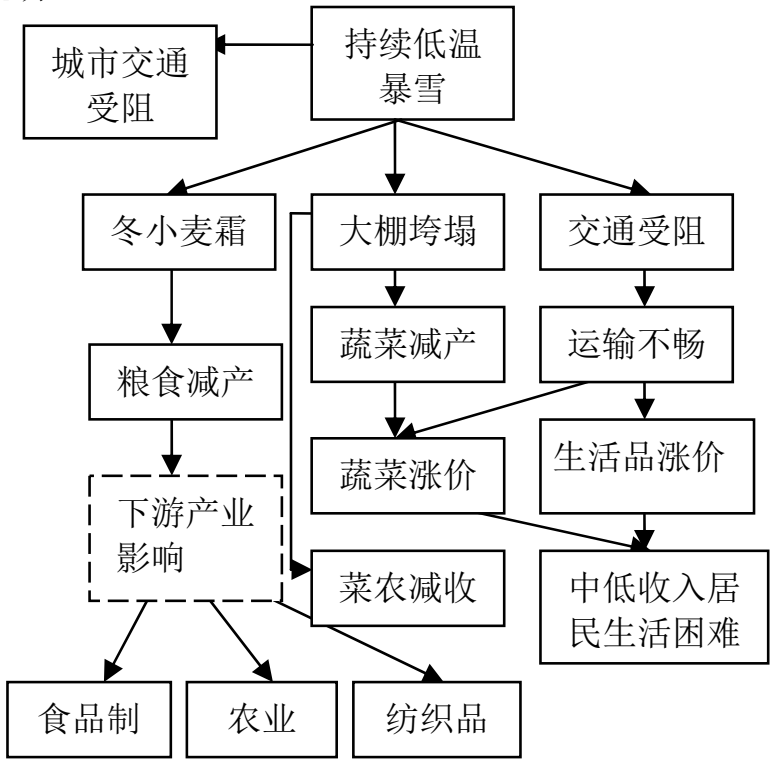

图 6. 2009 年华北雪灾风险扩散的识别

1) 冬小麦霜冻与粮食减产的关系

表 1 为从气象和农业部门收集的近年来冬小麦 霜冻与产量的资料。 
表 1. 冬小麦霜冻年份的产量与减产率

\begin{tabular}{ccccc}
\hline 年份 & $\begin{array}{c}\text { 温度 } \\
\text { (摄氏度) }\end{array}$ & $\begin{array}{c}\text { 单产 } \\
\text { (公厅/亩) }\end{array}$ & $\begin{array}{c}\text { 平均单产 } \\
\text { (公斤/亩) }\end{array}$ & $\begin{array}{c}\text { 减产率 } \\
(\%)\end{array}$ \\
2000 & -5.1 & 362.3 & & -3.49 \\
2001 & -4.2 & 359.1 & & -4.34 \\
2003 & -5.5 & 371.8 & 375.4 & -0.96 \\
2004 & -4.8 & 376.2 & & 0.22 \\
2009 & -7.2 & 363.6 & & -3.14 \\
\hline
\end{tabular}

鉴于资料样本少，采用信息扩散技术建立冬小 麦霜冻与产量的关系, 得到冬小麦霜冻与粮食减产 的关系如表 2 所示。

表 2. 冬小麦霜冻与减产的模糊关系

减产 $5 \%$ 减产 $4 \%$ 减产 $3 \%$ 减产 $2 \%$ 减产 $1 \%$ 减产 $0 \%$

\begin{tabular}{lllllll}
-10 度 & 0.233 & 0.232 & 0.23 & 0.226 & 0.222 & 0.215 \\
-9 度 & 0.404 & 0.405 & 0.403 & 0.399 & 0.393 & 0.386 \\
-8 度 & 0.617 & 0.619 & 0.618 & 0.615 & 0.61 & 0.603 \\
-7 度 & 0.828 & 0.831 & 0.831 & 0.83 & 0.827 & 0.822 \\
-6 度 & 0.973 & 0.976 & 0.977 & 0.977 & 0.976 & 0.974 \\
-5 度 & 1 & 1 & 1 & 1 & 1 & 1 \\
-4 度 & 0.894 & 0.891 & 0.888 & 0.887 & 0.886 & 0.886 \\
-3 度 & 0.692 & 0.687 & 0.682 & 0.679 & 0.677 & 0.676 \\
-2 度 & 0.463 & 0.456 & 0.451 & 0.447 & 0.444 & 0.442 \\
-1 度 & 0.266 & 0.261 & 0.256 & 0.253 & 0.25 & 0.248 \\
0 度 & 0.131 & 0.128 & 0.125 & 0.122 & 0.12 & 0.119 \\
\hline
\end{tabular}

\section{2)粮食减产对其他相关行业的关系}

依据石家庄统计局的投入产出分析表中各行业 对农业的直接消耗系数 ${ }^{[11]}$, 可知当地的农业对食品 制造及烟草加工业、农业、纺织业、造纸印刷及文 教用品制造业等行业的贡献较大（表 3)。

由于篇幅所限, 其他风险扩散的关系建立过程 见文献 ${ }^{[12]}$, 这里不再赘述。

\section{4. 基于风险扩散的系统风险计算}

本案例中气象致灾力有三个输入条件, 即: \{温 度, 雪量, 致灾因子发生的概率 $\}$, 下面以输入条件 $\{-$ 6 度, $30 \mathrm{~mm}, 0.9\}$ 为例, 说明主要节点风险扩散计 算的过程。
表 3. 2002 年石家庄市投入产出表中 8 个 主要行业对农业的直接消耗系数

\begin{tabular}{|c|c|c|}
\hline $\begin{array}{l}\text { 序 } \\
\text { 号 }\end{array}$ & 行业 & $\begin{array}{l}\text { 直接消耗 } \\
\text { 系数 }\end{array}$ \\
\hline 01 & 食品制造及烟草加工业 & 0.243 \\
\hline 02 & 农业 & 0.151 \\
\hline 03 & 纺织业 & 0.089 \\
\hline 04 & 造纸印刷及文教用品制造业 & 0.057 \\
\hline 05 & 木材加工及家具制造业 & 0.055 \\
\hline 06 & 住宿及餐饮业 & 0.038 \\
\hline 07 & 交通运输及仓储业 & 0.033 \\
\hline 08 & 服装皮革羽线及其制品业 & 0.029 \\
\hline
\end{tabular}

\section{1) 霜冻对粮食减产的风险}

根据霜冻与粮食减产的模糊关系, 根据公式 (4) 进行模糊推理, 当出现 -6 度时, 粮食减产率 约为 $2(\%)$, 对应的风险值为 0.977 。那么, 由严重 霜冻风险 (-6 度) 为 0.9 导致的粮食减产 $2 \%$ 的风险 是 $0.9 \times 0.977=0.879$ 。

\section{2）计算暴雪对蔬菜大棚垮塌的风险}

采用公式 (4) 进行模糊推理, 当降雪量 $30 \mathrm{~mm}$ 时, 由暴雪对蔬菜大棚垮塌率的关系可求得大棚的 垮塌率为 $23(\%)$, 从模糊关系中取接近的 $24 \%$, 即暴 雪 $30 \mathrm{~mm}$ 造成大棚垮塌率 $24 \%$ 的可能性最大, 对应的 风险值为 0.8 , 则大棚垮塌 $24 \%$ 的风险为: $0.9 \times$ $0.8=0.72$ 。

同理, 可以计算其他节点的风险值, 得到低 温、暴雪的风险。

风险的情景表示能够给出风险的直观表达，表 4 给出了一般情况下的风险等级说明。

表 4. 风险等级说明

\begin{tabular}{|c|c|c|c|}
\hline $\begin{array}{l}\text { 风险 } \\
\text { 级别 }\end{array}$ & $\begin{array}{l}\text { 颜色 } \\
\text { 标志 }\end{array}$ & 风险值区间 & 用语 \\
\hline 红色 & & $0.8<\mathrm{P} \leqslant 1$ & 将发生 \\
\hline 橙色 & & $0.7<\mathrm{P} \leqslant 0.8$ & 极有可能发生 \\
\hline 黄色 & & $0.6<\mathrm{P} \leqslant 0.7$ & 很可能发生 \\
\hline 蓝色 & & $0.5<P \leqslant 0.6$ & 可能发生 \\
\hline 灰色 & & $P \leqslant 0.5$ & 不提示 \\
\hline
\end{tabular}

风险扩散结果的情景表示如图 7 所示。 


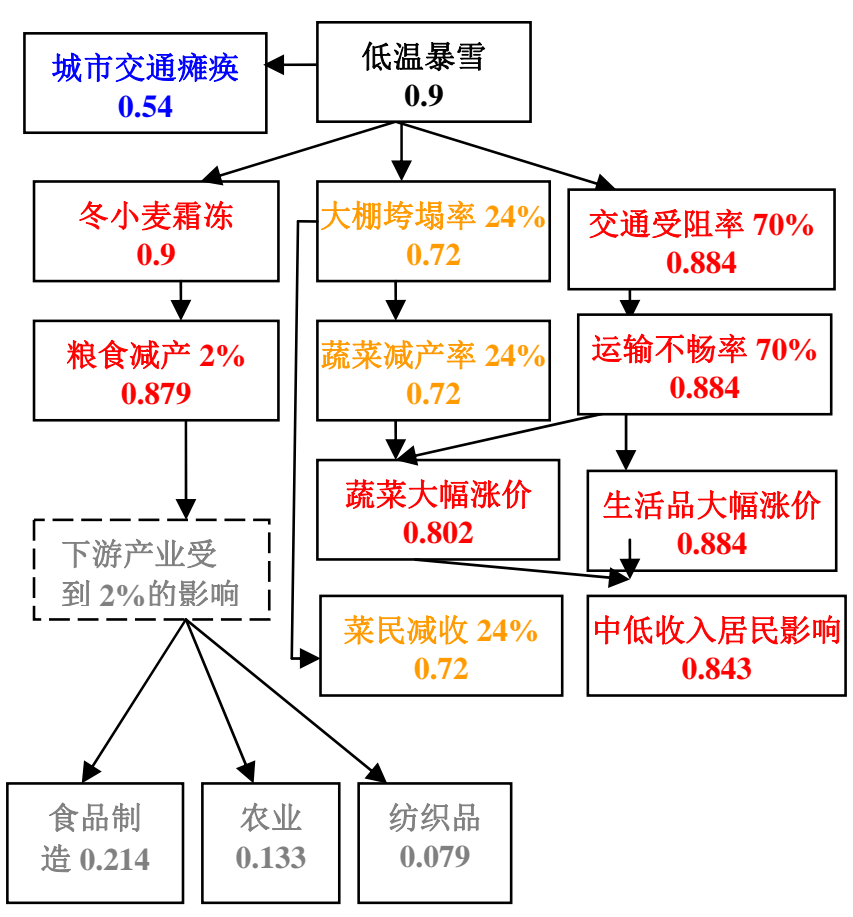

图 7. 2009 年暴雪的风险情景表示

由图 7 可知, 如果降雪量为 $30 \mathrm{~mm}$ 、最低气温 达到-6 度, 则将会对以下方面带来影响:

（1）暴雪将使冬小麦发生霜冻, 并导致夏粮减 产 2\%; 降雪将极有可能导致 24\%的温室大棚垮塌, 造成设施蔬菜减产 $24 \%$, 进而将使蔬菜价格大幅上 涨;

（2）暴雪将使公路交通受阻率达到 70\%, 由于 运输不畅将导致生活日用品价格大幅上涨，蔬菜和 日用品价格的上涨将对中低收入的居民造成生活支 出增加;

（3）暴雪还可能造成城市交通瘫疾, 综合生活 日用品和蔬菜价格的上涨因素, 将对部分居民生活 造成短时性的困难。

\section{5. 结论}

本文对气象灾害风险扩散的机理进行了研究, 得到以下结论:

1) 提出了气象灾害风险扩散的概念模型。气象 灾害风险扩散是气象致灾力作用于承灾体后, 由于 经济社会的系统性以及承灾体间的关联性, 致使对 其他承灾体产生的波及效应。

2）提出了气象灾害风险扩散的分析计算模型。 基于气象灾害风险扩散的概念模型, 提出了风险扩 散网络拓扑结构的层次分析数学算法, 给出了风险
扩散关系、风险扩散计算和节点风险融合的一般计 算处理方法。

3）开发了气象灾害风险扩散的仿真模拟系统。 根据气象灾害风险扩散的分析计算模型，建立了一 个可视化的风险分析仿真工具，以人机交互的方式 实现了风险扩散网络结构的构建、风险扩散的计 算。以 2009 年初冬的华北暴雪为例进行了实际案例 风险扩散的分析，给出了具有风险预警提示的风险 情景表示图, 进而验证了风险扩散分析理论与技术 方法的合理性。

\section{参考文献}

[1] 国家气候中心,2008 年中国气候公报.北京：中 国气象局，2009.

National Climate Center, 2008 China Climate Report, CMA, 2009.

[2] 国家气候中心.2009年中国气候公报. 北京: 中 国气象局, 2010.

National Climate Center, 2009 China Climate Report, CMA, 2010.

[3] 国家气候中心.2010 年中国气候公报.北京：中 国气象局, 2011.

National Climate Center, 2010 China Climate Report, CMA, 2011.

[4] Roadmap for Adapting to Coastal Risk. http://www.csc.noaa.gov/rvat/rvat.html

[5] KEN GRANGER. Quantify Storm Tide Risk in Chains. Natural Hazards, 2003,30(1): 165-185.

[6] 刘希林.区域泥石流灾害风险评价研究.自然灾害 学报,2000,9(1):54-61.

Liu xilin. Regional risk assessment on debris flow. Journal of Natural Disasters, 2000,9(1):54-61.

[7] Zhang jiquan. Risk assessment of drought disaster in the mainze-growing region of Songliao Plain, China. Agriculture Ecosystems \& Environment, 2004, 102(2):133-153.

[8] ICSC A Science Plan for Integrated Research on Disaster Risk. Paris,2008.

[9] 路踪, 魏一鸣, 范英等. 灾害对国民经济影响的 定量分析模型及其应用.自然灾害学报, 2002,

Vol.11 No.3,15-20.

Lu zong, Wei yiming, Fan ying. Quantitatively analytic model for the impact of natural disaster on national economy. Journal of Natural Disasters, 2002, Vol.11 No.3,15-20.

[10] 林均岐, 钟江荣.地震灾害产业关联损失评估.世 界地震工程, 2007 Vol.23 No.2, 37-41.

Lin junqi, Zhong jiangrong. Assessment of 
earthquake induced production sections-related

loss. World Earthquake Engineering, 2007 Vol.23

No.2, 37-41.

[11] 石家庄市统计局.2002 年度石家庄市投入产出表.

石家庄：石家庄市统计局, 2005.

Shi Jia Zhuang Statistical Bureau .2002 Shi Jia

Zhuang Input-Output Table. Shi Jia Zhuang: Shi

Jia Zhuang Statistical Bureau, 2005.

[12] 郭树军.气象灾害风险扩散机理研究及其在气象 灾害风险评估中的应用, 北京师范大学博士学 位论文, 2011.

Guo shujun, The Meteorological Disaster Risk

Diffusion Study and Application on

Meteorological Disaster Risk Assessment, $\mathrm{PhD}$

thesis, Beijing Normal University, 2011. 